\title{
Electric Field Dependence of Modulation in Multilayer InAs Quantum Dot Waveguides
}

\author{
Imran B. Akca ${ }^{1}$, Aykutlu Dana ${ }^{2}$, Atilla Aydinli ${ }^{2}$, Marco Rossetti ${ }^{3}$, Lianhe Li $^{3}$, Andrea Fiore ${ }^{3}$, N. Dagli ${ }^{4}$ \\ 1. Material Science and Nanotechnology Program, Bilkent University, Ankara, Turkey \\ 2. Material Science and Nanotechnology Program and Physics Department, Bilkent University, Ankara, Turkey \\ 3. Institute of Quantum Electronics and Photonics, Ecole Polytechnique Fédérale de Lausanne EPFL, CH-1015 Lausanne, Switzerland \\ 4. ECE Department, University of California at Santa Barbara, California, USA
}

Semiconductor quantum wells and quantum dots are known to be good candidates for switching and modulation devices in photonic integrated circuits [1]. In this regard, we characterized the electro-absorption and electro-optic properties of multilayer InAs quantum dot waveguides at 1309 and $1515 \mathrm{~nm}$.

We worked on three layers (3QDs) of quantum dots with the lengths of $1,1.3,1.6 \mathrm{~mm}$. The samples are grown by molecular beam epitaxy. The active region is formed by three layers of self-assembled InAs QDs, which are covered by a 5-nm $\mathrm{In}_{0.15} \mathrm{Ga}_{0.85} \mathrm{As} \mathrm{QW}$ and separated from each other by a 40-nm GaAs spacer layer. The areal dot density of our lens-shaped QDs is $3 \times 10^{10} \mathrm{~cm}^{-2}$. For each sample lasing is peaked at nearly $1285 \mathrm{~nm}[2]$.

Measurement of the electro-absorption and electro-optic coefficients at $1.3 \mu \mathrm{m}$ was carried out by coupling light from a tunable laser (Santec TSL-320) onto one end of the waveguide with a lens shaped fiber. At $1.5 \mu \mathrm{m}$, Santec Tunable LD Light Source TSL-520 was used. A controlled DC voltage source was used to apply 0 to 20 Volts reverse bias to the samples. At each voltage level, the transmission through the device is recorded as a function of wavelength and voltage.
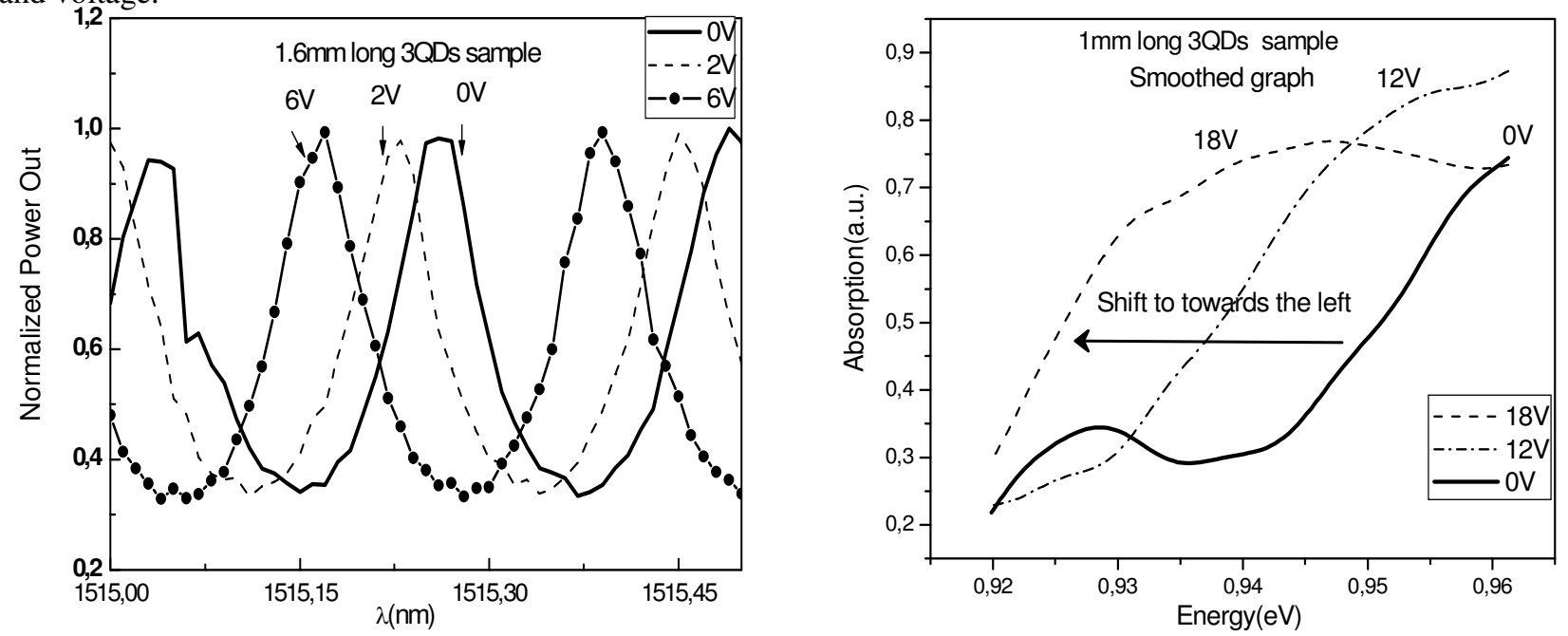

Figure 1 (a) Voltage dependency of FB resonances of 1.6mm long 3QDs sample. (b) Absorption versus energy graph of the $1 \mathrm{~mm}$ long 3QDs sample. The absorption spectra blue shifts under the applied voltages.

The average values for linear electro-optic coefficients $\left(\mathrm{r}_{41} / \Gamma\right)$ of 3QDs samples are given as $7.7 \times 10^{-13} \mathrm{~m} / \mathrm{V}$ which is an enhancement compared to the bulk GaAs [1]. As a matter of fact examination of Fig. 1a reveals that full on/off modulation is possible for $1.6 \mathrm{~mm}$ long $3 \mathrm{QD}$ sample using $6 \mathrm{~V}$. This corresponds to less than $1 \mathrm{~V}$-cm modulation efficiency. In other words using this modulator as the arms of a push pull driven Mach-Zehnder modulator, less than 1 $\mathrm{V}$ drive voltage would result for $1 \mathrm{~cm}$ long arms. We have also observed that absorption spectra of the samples blue shift with the applied voltage. We obtained the largest blue shift at the largest applied voltage of 18 Volts. The absorption spectrum is shown in Fig.1b.

In conclusion, we have observed the low voltage modulation in InAs quantum dot waveguides. We have measured the electro-optic coefficient in multilayer quantum dot structures far away from resonance and obtained an enhancement compared to bulk GaAs. Electro-absorption measurement results suggest that these waveguides are good candidates for use in electro-absorption modulators such as Mach-Zehnder devices.

This project is supported by Turkish Scientific and Technical Research Council (TUBITAK); Grant No: 103T115.

References:

[1]. L. Davis, K. Ko, W. Li, H.Sun, Y. Lam, T. Brock, S. Pang, and P. Bhattacharya, "Photoluminescence and electro-optic properties (25-35 nm diameter) quantum boxes", Appl. Phys. Lett., vol. 62, No.22, May 1993

[2]. A. Markus, J. X. Chen, C. Paranthoen, and A. Fiore," Simultaneous two-state lasing in quantum-dot lasers," Applied Physics Letter, vol. 82, No. 12, March 2003. 